\title{
Real Time Services for Cloud Computing Enabled Vehicle Networks
}

\author{
${ }^{1}$ G.sasikala, ${ }^{2}$ Dr.A.R. Deepti \\ Sr.Asst.Professor MCA department Reva Institution of Technology \& Management Banglore, India \\ Associate Professor, MCA department Acharya Institute of Technology Banglore, India
}

\begin{abstract}
Cloud computing technique is gaining more and more popularity recently. It can be applied to the vehicle applications to ensure real time performance as well as to improve accuracy and comfort degree for drivers. In this paper, we propose our novel vehicle cloud architecture which includes device level, communication level and service level. Each of these levels is explained in further detail with flow chart and taxonomy definition. Some innovative and real time vehicle cloud services are introduced to show the wide potential applications of vehicles and some discussion about research challenges, context classification is also provided.
\end{abstract}

Keywords- Vehicle cloud; Cloud computing, Real time service, Body area network, Healthcare

\section{Introduction}

With the fast development of automotive industry as well as Information and Communication Technology (ITC), our daily lives have been largely influenced and people tend to spend more and more time relevant to vehicles. It can be foreseen that the next generation transportation system will become a more powerful system by utilizing existing ommunication, network and computer infrastructure.

Currently one of the challenging issues to the road transportation system is the traffic congestion, which causes huge amount of financial and human life cost. In the United States alone, there is a loss of $\$ 78$ billion with 4.2 billion lost hours and 2.9 billion gallons lost of wasted gasoline in 2007 [1], not to mention about car accident with human casualty

Intelligent Transportation System (ITS) [2-4] has been developed to monitor traffic information, to reduce car accident, to alleviate workload of drivers and to improve their comfort degree. Usually, GPS-based devices, video camera, as well as other road side units (RSU) and road side infrastructure (RSI)

are utilized in parallel with ITS deployment.

In fact, vehicle is a very complex integrated system with mechanical system, computing and communication systems. On the one hand, all in-car vehicle resources (like CPU, memory, power, and communication units) need harmonious scheduling to achieve optimal efficiency. Also the sensor and actuator devices need to closely work with each other. On the other hand, each vehicle needs to cooperate with other vehicles or RSI in a vehicle-to-vehicle (V2V) or vehicle-toinfrastructure (V2I) manner in order to share and utilize external resources in a more effective way.

To facilitate the interaction between vehicle drivers and outside car world, a novel vehicle cloud (VCloud) architecture with real time services is proposed in this article. The concept of cloud computing (CC) is presented from economical point

of view and the key idea is to rent the software, platform and services rather than to buy them [5]. There are three types of cloud computing services, namely 1) Infrastructure as a Service (IaaS) where cloud providers provide their customers with storage, processing and network resources; 2) Platform as a

Service (PaaS) where the development tools are hosted in the cloud and accessed through a browser; 3) Software as a Service (SaaS) where the provider provide customers with application services in a pay-as-you-go manner.

The contribution of this article includes: 1) we propose a novel three tier V-Cloud architecture with detail explanation of each tier; 2) we provide detailed analysis and discussion about some sub-tier issues such as body area sensor network (BASN), context-aware middleware module etc. The flow chart and

relevant taxonomy if also given; 3 ) we present some innovative and real time services for future cloud vehicle applications

\section{Related Work}

In recent years, vehicles are equipped with smart phone devices with more powerful sensing, communication and processing capabilities. In [6] the authors the authors propose a VTrack system by using mobile phone in order to improve energy efficiency and sensor unreliability. They use hidden 
Markov model based mapping scheme and Viterbi decoding scheme to estimate travel time and the simulation results validate the performance of their VTrack system. The authors in [7] present a cooperative transit tracking method with the aid of built-in sensors inside the smart phone. An accelerometer based activity recognition algorithm is proposed to decide user riding behavior and another method for tracking underground vehicles is also given. In order to monitor road traffic, the authors in [8] propose a novel traffic control system based on their new design methodology and WSN.

How to provide real time services to mobile vehicles is always a research challenge. In [9] the authors develop a mobile sensing system for cyclist named BikeNet based on wireless sensor network. Various types of sensors are equipped on the bicycle to form a bicycle area network (BAN) and cyclist can share their real time data and experience by updating the common repository. In [10] the authors present the design, implementation and evaluation of a mobile vehicle system named ParkNet. With the aid of GPS device and ultrasonic rangefinder, real time parking statistics can be obtained with good ccuracy. The authors in [11] make a review of using 4 th generation $(4 \mathrm{G}$ ) wireless technology in cars and present the concept of mobile $4 \mathrm{G}$ car. A practical application platform is provided to illustrate some attractive services in the future

From system architecture point of view, the authors in [12] present a new navigation architecture for vehicles with detailed explanation of each sub-layers. The middleware system therein can enable functionalities such as perception, localization, mapping, real time motion and tracking etc. In [13] the authors present a smart car sensor network platform based on the OSGi framework and AMI-C standards. An architecture named SCSN is proposed with detailed hardware design and implementation.

There is some work which combines cloud computing concept with vehicle applications recently. The authors in [14] present the concept of autonomous vehicular cloud (AVC) with application scenarios like planned evacuation. The set up process of AVC is explained in detail and some AVC esearch challenges are also provided. In [15] the authors provide some survey work about the platform-as-a-service (PaaS) public cloud requirements with target application in a mobile and interactive environment like a car. In [16] the authors first make a comparison between existing on-demand bus system and the innovative on-demand bus system with cloud computing support technology. The cloud computing bus system is simulated based on developed calculation algorithm. The authors in [17] propose the concept of vehicular cloud computing (VC2) with special emphasis on underutilized vehicle resource sharing like computing, internet connectivity and storage etc.

The main difference between our work and those above is that: 1) we propose a novel three tier vehicle cloud architecture from device, communication and service level; 2) we give detailed explanation of submodules such as in-car body area sensor network, context aware middleware as well as some flow chart and taxonomy definition etc; 3 ) we provide several

customized and innovative cloud vehicle services like driver healthcare monitoring and in-car resource sharing services etc

\section{A. General Architecture}

\section{Our Three-Tier Vehicle Cloud Architecture}

With the aid of modern technologies such as MEMS, wireless communication techniques, Internet and cloud computing etc, we can imagine that future road transportation system will be more powerful, intelligent, flexible and convenient for both drivers and administrative centers

In Figure 1, we propose our novel three-tier V-Cloud architecture from functioning point of view. By integrating the cloud computing technique, we aim to provide to the users: 1) real time services; 2) sharing and cheap services; 3 ) more accurate general services; 4) customized services

As is shown in Figure 1, there are various devices in the tier-1 module ranging from sensors, actuators, GSP, smart phone and pc-based devices. It is worth mentioning that many of these tiny devices can form a body area sensor network (BASN) within a short range. Some of the devices may be equipped with the car while many others may be hand-held devices or attached on the driver to monitor the physical health information. 


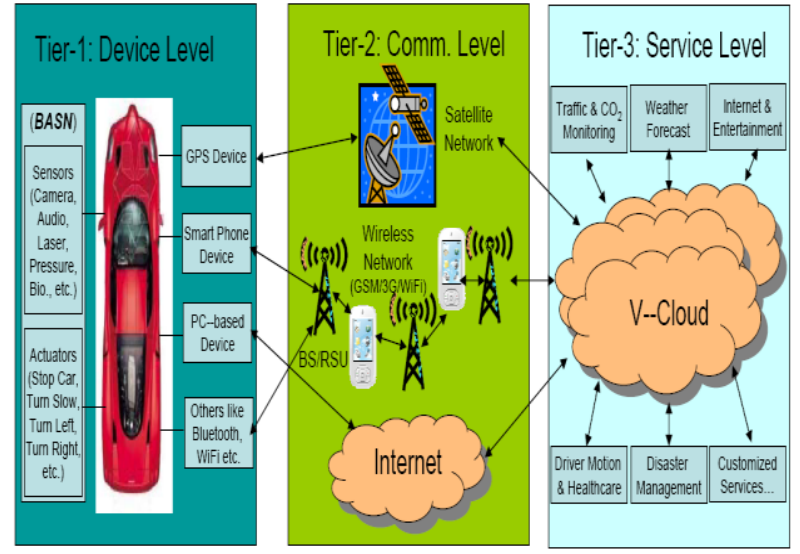

Figure 1. Three tier V-Cloud architecture

In tier-2, we classified the wireless communication into 3 groups: 1) communication to satellite network via GPS devices; 2) communication to $\mathrm{GSM} / 3 \mathrm{G} / \mathrm{WiFi}$ wireless networks via smart phone or other devices; 3) communication to the Internet via PC or navigation devices. It is worth noting that the communication with base station (BS) or road side unit (RSU) is part of group 2.

In tier-3, we can the core service module which is enabled by cloud computing technique. Here, we just list several representative services such as road traffic monitoring, $\mathrm{CO} 2$ pollution detection, Internet access, Entertainment, driver mood \& health monitoring etc. Many other customized services like parking, dining, reminding can also be developed based on driver's requirement or preference.

\section{B. Tier-1: Device Level}

The trend of car development is shifting from improving driver's safety to increasing their comfort and convenience degree. Consequently more and more tiny and smart devices have been deployed inside the vehicles or even attached to the drivers in order to provide real time measurement and protection services. These devices can communicate with each other in a self-organized manner and form a body area sensor network (BASN) through multi-hop manner.

Figure 2 shows the flow chart of BASN from sensor layer to actuator \& output layer in Tier-1. On the bottom sensor layer, raw data like temperature, pressure, image and biomedical information can be collected and then stored in a repository for future processing in the upper layer. In the repository, raw data can be further classified into low level context (like temperature, pressure) and high level context (like gesture, activity) based on pre-processing techniques. On knowledge processing layer, low level and high level context will be formally processed based on techniques like data mining, reasoning, k-means clustering etc. Finally, some outputs will be delivered through the context-aware middle layer to the upper actuator layer and some actions will be taken like stop the car, turn slow and send alarm signals to the driver. In the mean time, security is a key module for all the layers in the flow chart and security issues will include privacy issues, authorization and authentication etc.

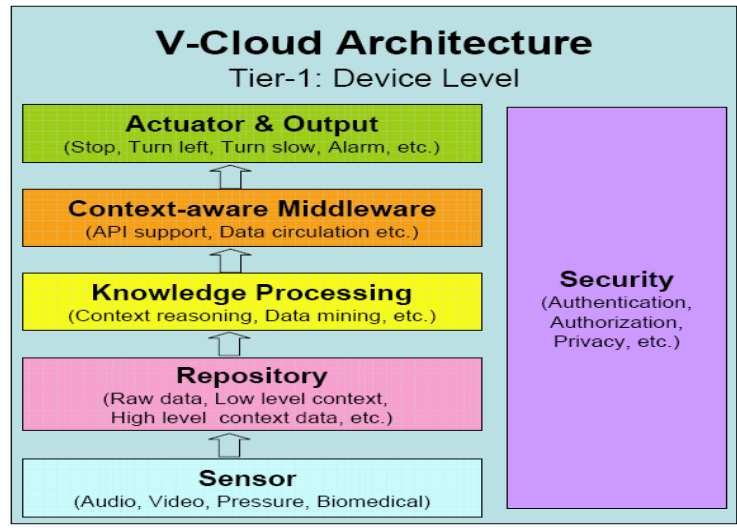

Figure 2. Flow chart of BASN in Tier-1

It is worth noting that not only the embedded sensors and actuators in the car can form a BASN but also some sensors attached to the drivers to collect physical information like EEG, ECG, heart rate etc. For example, sensors can collect driver's physical information (e.g. blood pressure, heart rate) and send 
all these data to a central repository or outside cloud for real time processing. After further reasoning and processing, if the driver's heart rate is higher than a pre-defined threshold, a warning message or sound will be sent or vibrating chair will be initiated to remind driver. The car will even get stopped under urgent situations.

On the right side of Tier-1 in Figure 1 are some portable devices with more flexibility and stronger capabilities. By using GPS localization technique, drivers can reduce their time on road and administrative centers can schedule vehicles (like bus, taxi) in a more efficient way especially during rush hours. Smart phone can largely enhance driver's comfort and convenience degree based on its strong sensing, processing and communication functions. More importantly, some customized application can be developed to interact with the Internet and other BASN devices like bio-sensors and video sensors etc. PC-based devices like in-car navigator can provide very friendly and interactive interface to users and they can enjoy various services such as road traffic monitoring, entertainment (audio, video, TV), Internet and resource sharing with other vehicles. Other devices like Bluetooth, WiFi can provide additional support and services to drivers based on their standard communication interface.

\section{Tier-2: Communication Level}

Due to the fast development of IT technology such as wireless communication, networking and signal processing techniques in the last decades of years, there are various alternatives for drivers to communicate with the outside world Figure 3 gives the classification of our proposed V-Cloud communication taxonomy in Tier-2. Based on the

communication objects, communication level in Tiere-2 can be further divided into in-car communication module, vehicle-tovehicle (V2V) communication module and vehicle-toinfrastructure (V2I) communication module. The tier-2 module

in Figure 1 mainly shows the V2I sub-module which includes the communication with satellite network, $3 \mathrm{G}$ wireless network and Internet. The in-car communication between different types of sensors, actuators and intelligent devices is mentioned in Section 3.2 with special focus on BASN.

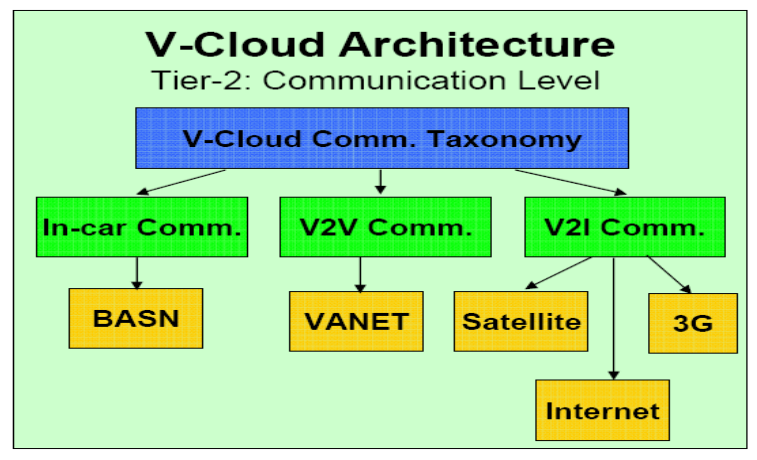

Figure 3. V-Cloud communication taxonomy in Tier-2

It is worth emphasizing that $\mathrm{V} 2 \mathrm{~V}$ communication paradigm can bring innovative and revolutionary applications to vehicle industry like vehicular ad hoc networks (VANET), V-Cloud enabled services and applications. This is because mobile vehicle has more powerful computing, processing, memory, bandwidth resources and they can share their resources in a self-organized and cooperative manner with elatively low cost. When there is no fixed infrastructure, the vehicles can selforganize and transmit their data in a hop-by-hop manner like the mobile ad hoc network (MANET). This kind of working paradigm is especially useful under applications such as battle field, real time and local traffic monitoring, emergent life saving and evacuation in remote areas.

\section{Tier-3: Service Level}

In Figure 4, the V-Cloud services of Tier-3 module in Figure 1 are further classified into context based,communication based and customized services. Some context based services include driver status monitoring, road pollution monitoring, car itself performance monitoring etc. This kind of context can also be deduced with output through knowledge processing and reasoning, as is explained in Section 3.2

Communication based services mainly include services which will be obtained through wireless communication techniques with outside car counterparts. We can see that context based services mainly focus on improving driver's safety and health while communication based services mainly focus on improving driver's comfort and convenience degree. 


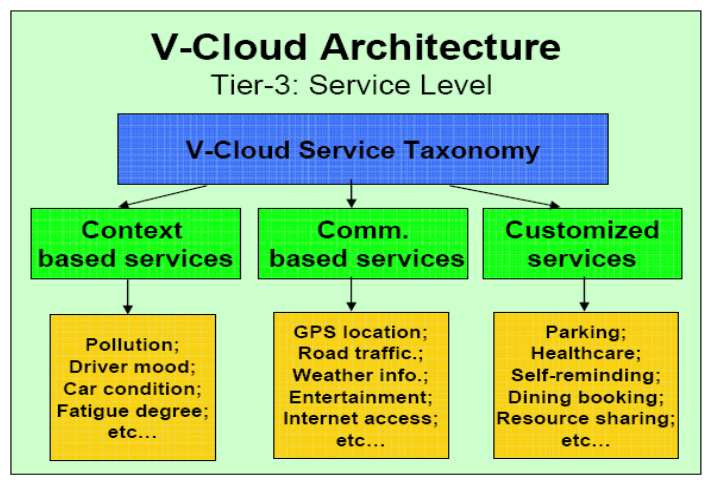

Figure 4. V-Cloud service taxonomy in Tier-3

Finally, some customized services can be developed for certain customers with special needs. For example, for those drivers who have heart disease or high blood pressure, some bio-sensor can be attached on them to measure their real time physical data and give them real time healthcare services. These healthcare services may include reminding them to take medicine, rest, warning them when they drive too fast or even help them to stop the car when necessary. In certain area when parking is a problem, drivers will prefer to pay s little investment on their navigator or smart phone so that they can easily find a suitable parking lot within a short time. More importantly, when the car is stopping with large amount of resources like memory/flash, power and computing capability, it can rent such resources to other vehicle users who is in need and is willing to pay some expense.

In summary, the three tier V-Cloud architecture can provide some innovative and real time services based on cloud computing techniques. It is worth noting that BASN with context-aware reasoning and knowledge processing techniques can largely improve drivers' safety, comfort and convenience

\section{Real Time Services Under Vehicle Cloud Architecture}

\section{A. Road Traffic Monitoring}

As one of the representative applications, road traffic monitoring is of vital importance to drivers. In order to reduce driving time, avoid unpleasant traffic accident as well as to reduce driving risks during severe weather, drivers will prefer to knowing the road traffic information beforehand and making decision by themselves or collaboratively with others.

Usually there are two kinds of methods to obtain road traffic information, namely centralized method and distributed method. For centralized method, drivers need to send inquiry to a central server or operator through smart phone or navigator

etc. Or they can listen to the radio transportation broadcast which is not desirable when drivers are only interested in certain roads.

Distributed method is gaining more and more popularity recently since it can broadcast traffic information in a real time manner to some interested groups or users. In one way, drivers near a traffic jam place can send broadcast or multicast message to their colleges (like taxi drivers) and friends or send

such information to the RSU/RSI so that the central

transportation center can make scheduling within a short time. Likewise drivers can send traffic information with good condition in a real time manner to their friends or to the RSU/RSI in order to reduce their driving time and to increase their convenience degree. It is worth noting that smart phone can play a very important role under such application scenarios since it has very powerful sensing, communication and internet access capabilities. Also, VANET is an important way to

realize the $\mathrm{V} 2 \mathrm{~V}$ information sharing service.

\section{B. Healthcare Monitoring}

Healthcare is a customized service provided to special group of drivers who might have certain diseases like heart disease, high blood pressure or other chronic diseases which may cause car accident. By adopting real time healthcare monitoring service, drivers can feel safer and more comfortable during driving and car accident rate as well as loss can be

largely reduced.

Healthcare monitoring service is an integrated service with combination of functions from Tier-1 to Tier 3 in Figure 1. First of all, some biomedical sensors like EEG, ECG, blood, glucose and pulse sensors are attached on the driver to measure his real time physical information. This information will be transmitted to some central devices like smart phone or other on board units with more powerful processing, memory and 
communication capabilities. Also, such information can be transmitted to certain outside cloud for further real time processing.

Once the measured value is above a pre-defined threshold value, some context-aware reasoning and knowledge processing techniques will be utilized to analyze such phenomenon and actions will be taken if necessary. These actions can include sending a reminding message to the smart phone or on board screen, sending an unpleasant voice to alert

driver, vibrating driver's chair or even stopping the car under emergent situations. As can be seen in Figure 1 and 2, contextbased knowledge processing and reasoning techniques play a vital role under such applications. The accuracy and real time performance of final output is guaranteed by cloud computing techniques either inside smart phone or from outside

It is worth emphasizing that many ustomized services for vehicle users can be implemented via smart phone or other on board units based on users' special needs. By integrating cloud computing techniques with networking and communication techniques etc, vehicle users can easily achieve their requirements with high comfort and convenience degree.

Taking parking as an example, on the one hand, drivers can send enquiry message to certain central parking service provider with cloud omputing technique support to obtain parking information. On the other hand, individual drivers can update parking lot information by using on board camera and rangefinder devices. They can send such information to a central server or outside cloud storage as they are driving so that later drivers can obtain parking lot information in a real time manner. Here we can see that screen devices like smart phone, navigator and notebook play an important role to obtain parking lot information.

Another example is resource sharing among vehicle users based on cloud computing concept. As is well know that cloud computing concept is started from economical point of view in order to save expense by renting resources rather than buying them. When vehicles are stopping at certain parking lot, their on board resources like storage, processing, network and entertainment resources are left unused or under utilization. On the other hand, some other vehicle drivers might need to use such resources to complete their task in a more efficient way. Therefore, such cloud computing inspired services can be fulfilled based on certain rental rate which both parties can accept.

\section{Discussion}

With the advent of smart phone in recent years, more and more intelligent vehicle services with cloud computing technique support can be easily implemented. This trend will become more obvious in the future as various types of sensors are mbedded nto the smart phone such as audio, video, accelerometer, GPS and biomedical sensors. Taking smart phone as an interface between human and internet as well as network, more and more customized services can be implemented in the future for vehicle users.

Security is always a challenge research for real time Vcloud services, as can be seen in Figure 2. It includes research issues ranging from smart phone and BASN communication, V2V authorization and authentication, vehicle drivers' privacy protection et

The context information can be classified into low level and high level context from layer point of view in Figure 2. It can also be classified into another three types, namely driver context, car context and road traffic context based on the research main body in Figure 2. Driver context mainly includes driver's health information, gesture, mood and activity etc. Car context mainly include power engine (e.g. engine status,accelerometer graph), security systems (e.g. air bag, safe belt,electronic lock) and comfort systems (e.g. navigator, air condition, entertainment).

\section{Conclusion}

Due to the fast development of ICT including smart phone, Internet, computer and wireless communication, the vehicle industry can be revolutionized and shifted to a new era. In this paper, we introduce the concept of cloud computing enabled real time vehicle services with special focus on customized services like healthcare, resource sharing, parking and dining etc. A three-tier V-Cloud architecture is proposed with detailed explanation about each sub-layer. Some flow chart as well as taxonomy definitions are also presented

\section{Acknowledgment}

This research work was also supported by the MKE (Ministry of Knowledge Economy), Korea, under the ITRC (Information Technology Research Center) support program supervised by the IITA (Institute of Information Technology Advancement) (IITA-2010-(C1090-1002-0003)) and by the basic science research program through the National Research Foundation (NRF) of Korea funded by the Ministry of Education, Science and Technology (2010-0016042). Professor Sungyoung Lee is the corresponding author 


\section{References}

[1] “Urban Mobility Report, Texas Transportation Institute, Texas A\&M University,” 2007.

[2] Intelligent Transportation Systems. http://www.its.dot.gov/.

[3] Francisco J. Martinez, Chai-Keong Toh, JJuan-Carlos Cano, Carlos T.Calafate, and Pietro Manzoni, Emergency Services in Future Intelligent Transportation Systems Based on Vehicular Communication Networks, IEEE Intelligent Transportation Systems Magazine, 2010, 2 (2),

[4] Panos Papadimitratos, Knut Evenssen,Vehicular Communication Systems: Enabling Technologies, Applications, and Future Outlook on Intelligent Transportation, IEEE Communications Magazine, Nov. 2009, pp.84-95.

[5] W. Kim, Cloud Computing: Today and Tomorrow, Journal of Object Technology, 8(1), January-February 2009, pp.65-72.

[6] A. Thiagarajan, L. Ravindranath, K. LaCurts, S. Madden, H.Balakrishnan, S. Toledo, J. Eriksson, Vtrack: accurate, energy-aware road traffic delay estimation using mobile phones, in: Int'l Conf. Embedded Networked Sensor Systems, 2009, 85-98.

[7] A. Thiagarajan, J. Biagioni, T. Gerlich, J. Eriksson, Cooperative transit tracking using smart-phones, in: Int'l Conf. Embedded Networked Sensor Systems, 2010, pp.85-98.

[8] Ivan Corredor, Ana-B Garcia, Jose-F Martinez, Pedro Lopez, Wireless Sensor Network-based system for measuring and monitoring road traffic, the 10th IEEE International Conference on Computer and Information Technology (CIT 2010), pp.13-19.

[9] S.B. Eisenman, E. Miluzzo, N.D. Lane, R.A. Peterson, G.S. Ahn, A.T. Campbell, The BikeNet mobile sensing system for cyclist experience mapping, in: Int'l Conf. Embedded Networked Sensor Systems, 2007, pp. 87-101.

[10] S. Mathur, T. Jin, N. Kasturirangan, J. Chandrashekharan, W. Xue, M. Gruteser, W. Trappe, ParkNet: drive-by sensing of road-side parking statistics, in: Int'l Conf. on Mobile Systems, Applications, and Services, 2010, pp.123-136.

[11] Jegor Mosyagin, Using 4G Wireless Technology in the Car, ICTON 2010.

[12] Gang Chen and Thierry Fraichard, A Real-Time Navigation Architecture for Automated Vehicles in Urban Environments, Proceedings of the IEEE Intelligent Vehicles Symposium Istanbul, 2007, pp.1123-1128.

[13] Pyungsun Park, Hongbin Yim, Heeseok Moon, Jaeil Jung, An OSGi Based In-Vehicle Gateway Platform Architecture for Improved Sensor Extensibility and Interoperability, the 33rd Annual IEEE International Computer Software and Applications Conference, 2009, pp.140-147.

[14] M. Eltoweissy, S. Olariu and M. Younis, Towards Autonomous Vehicular Clouds, Proc. AdHocNets, Victoria, BC, August 2010.

[15] David Bernstein,Nino Vidovic,Sohrab Modi, A Cloud PAAS for High Scale, Function, and Velocity Mobile Applications, 2010 the fifth International Conference on Systems and Networks Communications, pp.117-123.

[16] K. Tsubouchi, H. Yamato, K. Hiekata, Innovative on-demand bus system in Japan, IET Intelligent Transport Systems, 2010 , pp.270 -279 .

[17] Stephan Olariu, Ismail Khalil, Mahmoud Abuelela. Taking VANET to the clouds . Int. J. Pervasive Computing and Communications, 2011, 